BESANÇON

\title{
Le radical de Kaplansky
}




\title{
Le radical de Kaplansky *
}

\author{
Karim Johannes Becher \\ Département de Mathématiques de Besançon, Faculté des Sciences et Techniques, \\ 16. Route de Gray, 25030 Besançon Cedex, France \\ E-mail: becher@math.univ-fcomte.fr
}

1. Introduction $\ldots \ldots \ldots \ldots \ldots \ldots \ldots \ldots \ldots \ldots \ldots \ldots \ldots \ldots \ldots \ldots$

2. Notations et rappels........................

3. Structure du radical et exemples ............... 5

4. Radical et formes quadratiques................8

5. Corps quasi-pythagoriciens .................. 10

6. Radical et extension du corps ................13

7. Un problème de réduction d'indice ............. 17

8. Agrandissement et réduction du radical .......... 19

Références ................................26

\section{Introduction}

Dans toute la suite, $F$ désigne un corps de caractéristique différente de 2 . Le radical de $F$, noté $R(F)$, est l'ensemble des éléments $x \in F^{\times}$tels que soit $x$ est un carré dans $F$ soit $F(\sqrt{x}) / F$ est une extension quadratique pour laquelle l'homomorphisme de norme $F(\sqrt{x})^{\times} \rightarrow F^{\times}$est surjectif. Il s'avère que $\mathrm{R}(F)$ est un sous-groupe de $F^{\times}$.

Cette notion de radical pour un corps a été introduite par I. Kaplansky lorsqu'il étudiait les corps qui admettent une seule algèbre de quaternions àà division [8]. Par la suite, elle a été étudiée dans un cadre général par C. M. Cordes, qui la baptisa radical de Kaplansky, en l'honneur de son inventeur [3]. Cordes remarque que, dans certains énoncés en théorie des formes quadratiques, on peut remplacer le groupe des carrés $F^{\times^{2}}$ par le radical $\mathrm{R}(F)$.

"Le présent travail fait partie de la thèse de doctorat de l'auteur, effectuée à l'Université de Franche-Comté à Besançon sous la direction de Mme Eva Bayer-Fluckiger et M. Detlev Hoffmann et soutenue le 25 Septembre 2001. L'auteur tient à remercier ses directeurs de thèse pour leur soutien ainsi que $M$. Jean-Pierre Tignol et $M$. David Leep pour les discussions instructives qu'il a partagé avec eux au sujet de cet article et les suggestions précieuses dont ils lui ont fait part. Il est également très reconnaissant envers M. Emmanuel Lequeu pour son aide quant à la rédaction. 
C'est le cas, par exemple, pour le lemme de Kneser qui affirme que sur un corps non réel toute forme anisotrope de dimension $n$ représente au moins $n$ classes de carrés ; Cordes montre alors que, pour $n \geq 2$, une telle forme représente au moins $n$ classes (entières) modulo le radical. En particulier, il obtient ainsi une estimation pour le $u$-invariant d'un corps non réel avec un nombre fini de classes de carrés.

On note $\sum F^{\times^{2}}$ le sous-groupe de $F^{\times}$formé par les sommes non nulles de carrés dans $F$. Une partie majeure des travaux ultérieurs sur le radical a été consacrée à la construction de corps $F$ de radical non trivial, i.e. où les deux inclusions $F^{\times^{2}} \subset \mathrm{R}(F) \subset \sum F^{\times^{2}}$, valables en général, sont strictes. C'est encore Cordes qui a donné le premier exemple d'un tel corps. M. Kula [12] et L. Berman [1], indépendemment l'un de l'autre, ont construit des exemples de corps de radical non trivial avec un nombre fini de classes de carrés.

Le présent travail a pour but de résumer et d'approfondir les connaissances sur le radical de Kaplansky et de fournir de nouveaux exemples.

Dans les sections 3 et 4, nous exposons un certain nombre de faits sur le radical et nous donnons des exemples simples de la forme qu'il peut prendre.

Les deux sections suivantes s'inspirent d'un travail de D. Kijima, M. Nishi et T. Iwakami ([9], [10] et [7]). Dans la section 5, nous étudions les corps dits quasi-pythagoriciens, dans lesquels toute somme de carrés appartient au radical. Le premier résultat sur le radical, établi par Kaplansky lui-même, s'inscrit dans ce contexte. Dans la section 6 , nous cherchons à mettre en relation les radicaux de deux corps dont l'une est extension de l'autre. Le cas d'une extension quadratique présente un intérêt particulier : les quotients respectifs, pour chacun des deux corps, du groupe multiplicatif par le radical sont reliés par un certain complexe dont l'exactitude a été étudiée par Kijima, Nishi et Iwakami.

Le comportement du radical par rapport aux formes quadratiques, étudié dans la section 4 , incite à regarder les liens entre radical et algèbres simples centrales, ce qui est fait dans la section 7 .

Dans [11], Kula développe la théorie des schémas de formes quadratiques qui lui permet, en particulier, d'obtenir des corps de radical non trivial et avec un nombre fini de classes de carrés. Cette approche suggère que, pour un corps $F$, la structure des formes quadratiques modulo le radical $\mathrm{R}(F)$ et le quotient $\mathrm{R}(F) / F^{\times^{2}}$ paramètrent la structure des formes quadratiques sur $F$ de façon indépendante. Aussi souhaiterait-on faire varier le radical par extension des scalaires tout en préservant la structure des formes quadratiques modulo le radical. La construction d'agrandissement du radical, développée dans cet esprit en dernière section, donne lieu à des exemples très généraux de corps de radical non trivial pour lesquels la structure des formes quadratiques modulo le radical est imposée. 


\section{Notations et rappels}

Nous utiliserons librement les définitions et résultats de base en théorie algébrique des formes quadratiques sur un corps de caractéristique différente de 2. Les références standards sont [13] et [18]. Les formes quadratiques dont il est question dans la suite sont toutes supposées non dégénérées et l'on dira souvent seulement "forme" pour "forme quadratique". Les formes quadratiques de dimension 2 sont aussi appelées formes binaires. Pour deux formes quadratiques $\varphi_{1}$ et $\varphi_{2}$ on note $\varphi_{1} \perp \varphi_{2}$ (resp, $\varphi_{1} \otimes \varphi_{2}$ ) leur somme orthogonale (resp. leur produit tensoriel) et l'on écrit $\varphi_{1} \cong \varphi_{2}$ pour signifier que $\varphi_{1}$ et $\varphi_{2}$ sont isométriques.

Soit $\varphi$ une forme quadratique sur $F$. Si $a_{1}, \ldots, a_{m} \in F^{\times}$sont les valeurs de $\varphi$ sur une base orthogonale alors on écrit $\varphi \cong\left\langle a_{1}, \ldots, a_{m}\right\rangle$. Pour un entier positif $n$ on note $n \times \varphi$ la somme orthogonale $\varphi \perp \cdots \perp \varphi$ ( $n$ fois). L'ensemble des éléments non nuls de $F$ représentés par $\varphi$ est noté $D_{F}(\varphi)$. Si $D_{F}(\varphi)=F^{\times}$alors on dit que $\varphi$ est universelle.

2.1 Lemme. Soit $\varphi$ une forme quadratique sur $F$ et $a \in F^{\times}$. Pour que $\varphi$ représente a il faut et il suffit qu'il existe une forme quadratique $\varphi^{\prime}$ sur $F$ telle que $\varphi \cong \varphi^{\prime} \perp\langle a\rangle$. De plus, dans ce cas on a l'égalité

$$
D_{F}(\varphi)=\bigcup_{x \in D_{F}\left(\varphi^{\prime}\right)} D_{F}(\langle x, a\rangle)
$$

Démonstration: C'est clair.

On note $\left\langle\left\langle a_{1}, \ldots, a_{n}\right\rangle\right\rangle$ la $n$-forme de Pfister $\left\langle 1, a_{1}\right\rangle \otimes \cdots \otimes\left\langle 1, a_{n}\right\rangle$. C'est en particulier une forme multiplicative de dimension $2^{n}$.

Si les formes quadratiques $\varphi_{1}$ et $\varphi_{2}$ sur $F$ sont Witt équivalentes alors on écrit $\varphi_{1} \doteq \varphi_{2}$. On désigne par $W(F)$ l'anneau de Witt de $F$ et par $I(F)$ son idéal fondamental. Pour $n \geq 1$ on écrit $I^{n} F$ pour sa $n$-ième puissance, au lieu de $(I(F))^{n}$. Le déterminant de $\varphi \cong\left\langle a_{1}, \ldots, a_{m}\right\rangle$ est la classe du produit $a_{1} \cdots a_{m}$ dans $F^{\times} / F^{\times^{2}}$. Il est noté $d(F)$. On appelle alors discriminant de $\varphi$ la classe $d_{ \pm}(\varphi):=(-1)^{\frac{m(m-1)}{2}} \cdot d(\varphi) \in F^{\times} / F^{x^{2}}$. Il ne dépend que de la classe de $\varphi$ dans $W(F)$. Ainsi le discriminant définit une application $d_{ \pm}: W(F) \rightarrow F^{\times} / F^{\times 2}$. De plus, sa restriction à $I(F)$ est un homomorphisme de groupes. Par abus de notation, nous regarderons souvent $d_{ \pm}(\varphi)$ comme un élément de $F^{\times}$, déterminé à un facteur carré près.

Nous notons $\operatorname{Br}(F)$ le groupe de Brauer de $F$. Sa loi de groupe est notée additivement. Soit $A$ une $F$-algèbre simple centrale. Le degré de $A$ est par définition la racine carrée de la dimension de $A$ sur $F$. On note $[A]$ la classe de $A$ dans $\operatorname{Br}(F)$ et on appelle exposant de $A$, noté $\exp _{F}(A)$, l'ordre de 
$[A]$ dans $\operatorname{Br}(F)$. L'indice de $A$, noté $\operatorname{ind}_{F}(A)$, est le degré de l'unique $F$ algèbre à division $D$ qui représente la même classe que $A$ dans $\operatorname{Br}(F)$. A est dite déployée lorsqu'elle représente la classe triviale dans $\operatorname{Br}(F)$, i.e. lorsque $A \cong \mathcal{M}_{n}(F)$ pour un $n \geq 1$.

On rappelle que, pour $a, b \in F^{\times}$, la $F$-algèbre de quaternions $(a, b)_{F}$ a pour forme norme la 2 -forme de Pfister $\langle\langle-a,-b\rangle\rangle$.

2.2 Lemme. Pour $a, b \in F^{\times}$les conditions suivantes sont équivalentes :

(i) La F-algèbre $(a, b)_{F}$ est déployée.

(ii) La 2-forme de Pfister $\langle\langle-a,-b\rangle\rangle$ est hyperbolique.

(iii) La forme quadratique $\langle 1,-a,-b\rangle$ est isotrope.

Référence : [13, Chap. III, Theorem. 2.7.].

2.3 Lemme. Pour $a, b \in F^{\times}$on a $D_{F}(\langle 1, a\rangle) \cap D_{F}(\langle 1, b\rangle) \subset D_{F}(\langle 1,-a b\rangle)$.

Démonstration : Soit $x \in D_{F}(\langle 1, a\rangle) \cap D_{F}(\langle 1, b\rangle)$. Il vient $-a,-b \in$ $D_{F}(\langle 1,-x\rangle)$ et ainsi $a b \in D_{F}(\langle 1,-x\rangle)$, d'où $x \in D_{F}(\langle 1,-a b\rangle)$.

Soit $K / F$ une extension de corps. L'extension de scalaires induit un homomorphisme canonique de groupes $\mathrm{Br}(F) \rightarrow \mathrm{Br}(K)$ ainsi qu'un homomorphisme canonique d'anneaux $W(F) \rightarrow W(K)$. Le noyau de $\operatorname{Br}(F) \rightarrow \operatorname{Br}(K)$ est noté $\operatorname{Br}(K / F)$ et appelé groupe de Brauer relatif de $K / F$. Pour une extension finie $K / F$, on note $N_{K / F}$ l'homomorphisme de norme $K^{\times} \rightarrow F^{\times}$.

2.4 Proposition. Soit $K:=F(\sqrt{a})$, avec $a \in F^{\times} \backslash F^{\times^{2}}$. Les homomorphismes d'inclusion $F^{\times} \hookrightarrow K^{\times}$et de norme $N_{K / F}: K^{\times} \rightarrow F^{\times}$donnent lieu $\grave{a}$ une suite exacte de groupes

$$
1 \longrightarrow\left\{F^{\times 2}, a F^{\times 2}\right\} \longrightarrow F^{\times} / F^{\times 2} \longrightarrow K^{\times} / K^{\times 2} \longrightarrow F^{\times} / F^{\times 2} .
$$

Référence : [13, Chap. VII, Theorem 3.4.].

2.5 Proposition (Principe de la Norme). Soient $K / F$ une extension quadratique, $\varphi$ une forme quadratique sur $F$ et $x \in K^{\times}$. Pour qu'il existe $c \in F^{\times}$tel que $c x \in D_{K}\left(\varphi_{K}\right)$ il faut et il suffit que $N_{K / F}(x) \in D_{F}(\varphi) \cdot D_{F}(\varphi)$.

Référence : [6, Norm Principle 2.13.].

Remarque : On utilisera le Principe de la Norme surtout dans le cas où $\varphi$ est une forme de Pfister; dans ce cas on a $D_{F}(\varphi) \cdot D_{F}(\varphi)=D_{F}(\varphi)$, puisque $\varphi$ est multiplicative. 
Une extension $K / F$ qui s'injecte dans une clôture quadratique de $F$ est appelée 2-extension. L'énoncé suivant est classique :

2.6 Lemme. Soit $K / F$ une 2-extension non triviale. Alors $K$ contient une extension quadratique de $F$.

Démonstration : Si l'on choisit un élément $x \in K^{\times} \backslash F^{\times}$, alors $F(x) / F$ est une 2-extension non triviale contenue dans $K / F$. On peut donc supposer que $K / F$ est finie. Soit $L / F$ une clôture galoisienne de $K / F$. Comme toute clôture quadratique de $F$ est une extension galoisienne de $F, L / F$ est également une 2-extension. On note $G$ le groupe de Galois de $L / F$ et $H$ le groupe de Galois de $L / K$. Alors $G$ est un 2-groupe et, comme l'extension $K / F$ est non triviale, on a $H \neq G$. On choisit un sous-groupe maximal $H^{\prime}$ de $G$ qui contient $H$. Alors $H^{\prime}$ est d'indice 2 dans $G$ (cf. [2, Chap. I, $\S 6$, Proposition 12]) et $L^{H^{\prime}}$, le sous-corps de $L$ fixé par $H^{\prime}$, est une extension quadratique de $F$ contenue dans $K$.

\section{Structure du radical et exemples}

Nous appelons radical de $F$ l'ensemble

$$
\mathrm{R}(F):=\bigcap_{a \in F^{\times}} D_{F}(\langle 1, a\rangle)
$$

Il vient de cette définition que le radical de $F$ est un sous-groupe de $F^{\times}$ et que l'on a les inclusions $F^{\times^{2}} \subset \mathrm{R}(F) \subset \sum F^{\times^{2}}$. Dans cette section, nous donnons des exemples qui illustrent les diverses positions possibles du radical $\mathrm{R}(F)$ par rapport à $F^{x^{2}}$ et $\sum F^{\mathrm{x}^{2}}$. Il convient d'abord de chercher des caractérisations équivalentes pour les éléments du radical.

3.1 Proposition. Pour $x \in F^{\times}$les conditions suivantes sont équivalentes :

(i) $x \in \mathrm{R}(F)$.

(ii) La forme $\langle 1,-x\rangle$ est universelle sur $F$.

(iii) Pour tout $a \in F^{\times}$on a l'égalité $D_{F}(\langle 1, a\rangle)=D_{F}(\langle 1, a x\rangle)$.

(iv) La $F$-algèbre $(x, y)_{F}$ est déployée quel que soit $y \in F^{\times}$.

(v) $\operatorname{Br}(F(\sqrt{x}) / F)=0$. 
Référence : Les équivalences $(i \Leftrightarrow i i \Leftrightarrow i v)$ sont mentionnées dans [3, p. 253].

Démonstration: Les conditions (i) et (ii) sont chacune équivalentes à ce que la forme $\langle 1,-x,-y\rangle$ soit isotrope quel que soit $y \in F^{\times}$, ce qui est, d'après (2.2), également équivalent à $(i v)$. De (2.3) on déduit l'égalité

$$
D_{F}(\langle 1, a\rangle) \cap D_{F}(\langle 1,-x\rangle)=D_{F}(\langle 1, a x\rangle) \cap D_{F}(\langle 1,-x\rangle)
$$

pour $a, x \in F^{\times}$, d'où l'implication $(i i \Rightarrow i i i)$. Pour la réciproque, il suffit de prendre $a:=-1$.

Sans hypothèse sur $x \in F^{\times}$, le groupe de Brauer relatif $\operatorname{Br}(F(\sqrt{x}) / F)$ consiste en les classes de $F$-algèbres de quaternions $(x, y)_{F}$, avec $y \in F^{\times}$. L'équivalence $(i v \Leftrightarrow v)$ s'ensuit.

3.2 Corollaire. Pour que le corps $F$ satisfasse $\mathrm{R}(F)=F^{\times}$il faut et il suffit que toute forme binaire sur $F$ soit universelle.

Un corps $F$ qui vérifie $\mathrm{R}(F)=F^{\times}$est non réel. De façon plus générale, on s'intéresse aux corps $F$ pour lesquels $\mathrm{R}(F)=\sum F^{\times^{2}}$. Ces corps, dits quasi-pythagoriciens, seront étudiés en section 5 .

3.3 Exemples. Dans les cas suivants le corps $F$ vérifie $\mathrm{R}(F)=F^{\times}$:

(i) F est un corps fini (de caractéristique impaire),

(ii) $F=k((X))$ où $k$ est un corps quadratiquement clos (avec $\operatorname{car}(k) \neq 2$ ),

(iii) $F=k(X)$ où $k$ est un corps algébriquement clos (avec $\operatorname{car}(k) \neq 2$ ).

En effet, dans les cas $(i)$ et $(i i), F$ est non réel avec $\left|F^{\times} / F^{\times^{2}}\right|=2$, donc il existe seulement deux formes binaires sur $F$ et elles sont universelles. Dans (iii) le corps $F$ possède la propriété $\mathcal{C}_{1}$ (voir [18, Chap. 2, § 15]) et ainsi toute forme binaire sur $F$ est universelle.

Il arrive souvent que le radical du corps est réduit au groupe des carrés. On dit que le corps $F$ est sans radical si $\mathrm{R}(F)=F^{\times^{2}}$.

3.4 Exemples. (1) Tout corps local est sans radical. En effet, sur un corps local $F$ il existe une $F$-algèbre de quaternions à division qui est déployée par toute extension quadratique de $F$ [13, Chap. IV, Lemma 2.14] ; par conséquent, on a $\operatorname{Br}(F(\sqrt{x}) / F) \neq 0$ pour $x \in F^{\times} \backslash F^{\times^{2}}$; il s'ensuit par l'implication $(i \Rightarrow v)$ de $(3.1)$ que $\mathrm{R}(F)=F^{\mathrm{x}^{2}}$.

(2) Tout corps pythagoricien est sans radical. C'est clair car pour un corps pythagoricien $F$ on a par définition $\sum F^{\times^{2}}=F^{\times^{2}}$ et ainsi les inclusions 
$F^{\times^{2}} \subset \mathrm{R}(F) \subset \sum F^{\times^{2}}$ sont des égalités. En fait, un corps est pythagoricien si et seulement s'il est quasi-pythagoricien et sans radical.

(3) Tout corps de nombres est sans radical. Pour le vérifier, on considère pour un corps de nombres $F$ ses complétés $F_{\wp}$ pour toutes les places $\wp$ sur $F$. Alors, de l'égalité $F_{\wp}^{\times}=F^{\times} F_{\wp}^{\times 2}$ on déduit par l'équivalence $(i \Leftrightarrow i i)$ de (3.1) que $\mathrm{R}(F) \subset \mathrm{R}\left(F_{\wp}\right)$. Or, $\mathrm{R}\left(F_{\wp}\right)=F_{\wp}^{\times 2}$ puisque $F_{\wp}$ est soit un corps local soit un corps réellement clos et ainsi pythagoricien. Un élément de $\mathrm{R}(F)$ devient donc un carré dans tout complété $F_{\wp}$; le "théorème du carré global" [14, p. 182] dit alors que cet élément est un carré dans $F$.

3.5 Proposition. Soit $F$ un corps complet pour une valuation discrète avec un corps résiduel de caractéristique différente de 2 et non quadratiquement clos. Alors $F$ est sans radical.

Démonstration : Soient $v$ la valuation pour laquelle $F$ est complet et $\pi$ une uniformisante pour $v$. Il suit de [13, Chap. VI, Proposition 1.9.] que $D_{F}(\langle 1, a \pi\rangle)=F^{\times^{2}} \cup a \pi F^{\times^{2}}$ pour tout $a \in F^{\times}$tel que $v(a)=0$. Comme le corps résiduel n'est pas quadratiquement clos il existe un élément $a \in$ $F^{\times} \backslash F^{\times^{2}}$ tel que $v(a)=0$. Il vient $\mathrm{R}(F) \subset D_{F}(\langle 1, \pi\rangle) \cap D_{F}(\langle 1, a \pi\rangle)=F^{\times^{2}}$, donc $\mathrm{R}(F)=F^{\times^{2}}$.

3.6 Corollaire. Si $F$ est un corps non quadratiquement clos alors $F((X))$ est sans radical.

On s'intéresse maintenant aux corps qui ne sont ni quasi-pythagoriciens ni sans radical. On dira que le corps $F$ est de radical non trivial lorsque $F^{\times^{2}} \subsetneq \mathrm{R}(F) \subsetneq \sum F^{\times^{2}}$. Pour les corps non réels cette définition cö̈ncide avec celle qui est donnée dans [3, p. 259]. Nous donnons un exemple d'une extension algébrique non réelle de $\mathbb{Q}$ avec 8 classes de carrés et de radical non trivial.

3.7 Exemple. Dans $\mathbb{Q}_{3}$ les entiers $-2,-5$ et 7 sont des carrés. $\mathbb{Q}_{3}$ contient donc le corps $\mathbb{Q}(\sqrt{-2}, \sqrt{-5})$, dans lequel 7 n'est pas un carré. Parmi les sous-corps de $\mathbb{Q}_{3}$ qui sont extension algébrique de $\mathbb{Q}(\sqrt{-2}, \sqrt{-5})$ et dans lesquels 7 n'est pas un carré, on choisit un corps $F$ maximal. Alors $F(\sqrt{7})$ est la seule extension quadratique de $F$ dans $\mathbb{Q}_{3}$. Les quatre classes de carrés de $\mathbb{Q}_{3}$ étant représentées par $1,2,3$ et 6 , il s'ensuit que les classes de $2,3,7$ forment une $\mathbb{F}_{2}$-base de $F^{\times} / F^{\times^{2}}$; en particulier $\left|F^{\times} / F^{\times 2}\right|=8$.

On déduit de $\mathbb{Q}_{3}^{\times}=F^{\times} \mathbb{Q}_{3}^{\times^{2}}$ que $\mathrm{R}(F) \subset \mathrm{R}\left(\mathbb{Q}_{3}\right)$. Comme $\mathbb{Q}_{3}$ est sans radical il vient $\mathrm{R}(F) \subset F^{\times} \cap \mathbb{Q}_{3}^{\times^{2}}=F^{\times^{2}} \cup 7 F^{\times^{2}}$. On calcule $2=3^{2}-7$, $3=(\sqrt{-2} \cdot \sqrt{-5})^{2}-7$ et $2 \cdot 3 \cdot 7=7^{2}-7$; on en conclut $D_{F}(\langle 1,-7\rangle)=F^{\times}$, d'où $\mathrm{R}(F)=F^{\times^{2}} \cup 7 F^{\times^{2}}$. 


\section{Radical et formes quadratiques}

Dans cette section, nous exposons plusieurs résultats qui éclairent le rôle joué par le radical de Kaplansky dans la théorie des formes quadratiques. En particulier, nous mettons en correspondance le radical avec l'idéal de l'anneau de Witt formé par les formes binaires universelles (4.4), et, pour tout élément $a \in \mathrm{R}(F)$, nous déterminons le noyau de l'homomorphisme canonique $W(F) \rightarrow W(F(\sqrt{a}))(4.5)$.

4.1 Lemme. Soit $\beta$ une forme binaire sur $F$. Pour que $\beta$ soit universelle $i l$ faut et il suffit que son discriminant $d_{ \pm}(\beta)$ appartienne à $\mathrm{R}(F)$. Si tel est le cas, alors $\beta \cong\left\langle 1,-d_{ \pm}(\beta)\right\rangle$ et pour toute forme quadratique $\varphi$ sur $F$ on a

$$
\varphi \otimes \beta \doteq \begin{cases}0 & \text { si } \varphi \text { est de dimension paire } \\ \beta & \text { si } \varphi \text { est de dimension impaire }\end{cases}
$$

$\beta$ soit universelle il faut et il suffit que $\left\langle 1,-d_{ \pm}(\beta)\right\rangle$ soit universelle, i.e. que $d_{ \pm}(\beta) \in \mathrm{R}(F)$.

Démonstration : La forme $\beta$ est semblable à $\left\langle 1,-d_{ \pm}(\beta)\right\rangle$. Pour que $\beta$ soit universelle il faut et il suffit que $\left\langle 1,-d_{ \pm}(\beta)\right\rangle$ soit universelle, i.e. que $d_{ \pm}(\beta) \in \mathrm{R}(F)$.

On suppose maintenant que $\beta$ est universelle. En particulier, $\beta$ représente 1 , d'où $\beta \cong\left\langle 1,-d_{ \pm}(\beta)\right\rangle$. Soit $\varphi$ une forme quadratique sur $F$. Alors $\beta \otimes \varphi$ est isotrope dès lors que $\operatorname{dim}(\varphi) \geq 2$. Si $\operatorname{dim}(\varphi)=2$ alors $\beta \otimes \varphi$ est semblable à une 2-forme de Pfister et, étant isotrope, elle est hyperbolique. Par conséquent, $\beta \otimes \varphi$ est hyperbolique si $\varphi$ est de dimension paire. Si $\varphi$ est de dimension impaire alors on écrit $\varphi \cong \varphi^{\prime} \perp\langle z\rangle$, avec un élément $z$ de $F^{\times}$et une forme de dimension paire $\varphi^{\prime}$, et on obtient $\varphi^{\prime} \otimes \beta \doteq 0$ et ainsi $\varphi \otimes \beta \doteq z \beta \cong \beta$.

4.2 Proposition (Cordes). Soient $a_{1}, \ldots, a_{n} \in F^{\times}$et $r_{1}, \ldots, r_{n} \in \mathrm{R}(F)$ avec $n \geq 2$.

(a) Les formes $\left\langle a_{1}, \ldots, a_{n}\right\rangle$ et $\left\langle a_{1} r_{1}, \ldots, a_{n} r_{n}\right\rangle$ représentent les mêmes éléments de $F^{\times}$.

(b) Si $n \geq 3$ alors $\left\langle a_{1}, \ldots, a_{n}\right\rangle$ est isotrope si et seulement si $\left\langle a_{1} r_{1}, \ldots, a_{n} r_{n}\right\rangle$ est isotrope.

(c) $\left\langle\left\langle a_{1}, \ldots, a_{n}\right\rangle\right\rangle \cong\left\langle\left\langle a_{1} r_{1}, \ldots, a_{n} r_{n}\right\rangle\right\rangle$. 
Référence : Les parties (a) et (b) proviennent de [3, Proposition 1 \& Corollary]. Une version plus générale de (c) se trouve dans [21, Theorem 2.4.].

Démonstration: Tous les énoncés s'obtiennent par récurrence à partir du cas où $r_{1}=\cdots=r_{n-1}=1$. On se place alors dans cette situation et l'on pose $a:=a_{n}, r:=r_{n}, \varphi:=\left\langle a_{1}, \ldots, a_{n-1}\right\rangle$ et $\pi:=\left\langle\left\langle a_{1}, \ldots, a_{n-1}\right\rangle\right\rangle$.

(a) Supposons que $\varphi \perp\langle a\rangle$ représente $x \in F^{\times}$. On peut alors écrire $\varphi \cong \varphi^{\prime} \perp\langle b\rangle$ où $b$ est un élément de $F^{\times}$tel que $x$ est représenté par la forme binaire $\langle b, a\rangle$ et $\varphi^{\prime}$ est une forme sur $F$. Comme $r \in \mathrm{R}(F)$, l'implication $(i \Rightarrow$ iii $)$ de $(3.1)$ donne $b x \in D_{F}(\langle 1, a b\rangle)=D_{F}(\langle 1, r a b\rangle)$ et ainsi $x \in$ $D_{F}(\langle b, a r\rangle) \subset D_{F}(\varphi \perp\langle a r\rangle)$. Cela montre l'inclusion $D_{F}(\varphi \perp\langle a\rangle) \subset$ $D_{F}(\varphi \perp\langle a r\rangle)$. Par symétrie, on a l'égalité.

(b) Soit $n \geq 3$. Si $\varphi \perp\langle a\rangle$ est isotrope alors on peut écrire $\varphi \cong \varphi^{\prime} \perp\langle-a\rangle$ avec $\varphi^{\prime}$ une forme non triviale. Comme $\langle-a$, ar $\rangle$ est universelle, $\varphi \perp\langle a r\rangle$ est isotrope. Par symétrie on a l'équivalence de l'énoncé.

(c) La forme $\pi \otimes\langle a,-a r\rangle$ est hyperbolique d'après (4.1). Il vient $a \pi \cong$ $a r \pi$ et ainsi $\pi \otimes\langle 1, a\rangle \cong \pi \otimes\langle 1, a r\rangle$.

4.3 Corollaire (Cordes). Pour toute forme quadratique $\varphi$ de dimension au moins 2 sur $F$, l'ensemble $D_{F}(\varphi)$ est l'union des classes de $F^{\times}$modulo $\mathrm{R}(F)$.

4.4 Proposition. L'application $\mathrm{R}(F) \rightarrow W(F)$ qui à un élément $x \in \mathrm{R}(F)$ associe la classe de la forme $\langle 1,-x\rangle$ est un homomorphisme de groupes. Son noyau est $F^{\times^{2}}$ et son image est l'idéal de $W(F)$ formé par les classes des formes binaires universelles.

Démonstration : Pour $x, y \in \mathrm{R}(F)$ la forme quadratique $\langle 1,-x, 1,-y\rangle$ est isotrope puisqu'elle contient des sous-formes binaires universelles. Il vient $\langle 1,-x, 1,-y\rangle \doteq \beta$ pour une forme binaire $\beta$ de discriminant $x y \in \mathrm{R}(F)$, donc pour $\beta \cong\langle 1,-x y\rangle$. Cela montre que l'application $\mathrm{R}(F) \rightarrow W(F)$ est un homomorphisme de groupes. Il est clair que son noyau est $F^{\times^{2}}$ et, en vertu de (4.1), que son image est un idéal, constitué des classes des formes binaires universelles.

4.5 Théorème. Soit $K=F(\sqrt{a})$ avec $a \in \mathrm{R}(F) \backslash F^{\times^{2}}$. Alors $\langle 1,-a\rangle$ est, à isométrie près, la seule forme quadratique anisotrope sur $F$ qui devienne isotrope par extension des scalaires à $K$. En particulier, $\langle 1,-a\rangle$ représente la seule classe non triviale dans le noyau de l'homomorphisme canonique $W(F) \rightarrow W(K)$. 
Démonstration : Comme $a$ n'est pas un carré dans $F$, la forme $\langle 1,-a\rangle$ est anisotrope sur $F$ et hyperbolique sur $K$. Supposons maintenant que $\varphi$ est une forme quadratique anisotrope sur $F$ telle que $\varphi_{K}$ est isotrope. Donc $\varphi$ contient une sous-forme binaire $\beta$ de discriminant $a[18,2.5 .1$. Lemma]. D'après (4.1), $\beta$ est universelle et isométrique à $\langle 1,-a\rangle$, car $a \in \mathrm{R}(F)$. Comme $\varphi$ est anisotrope, il vient $\varphi \cong \beta \cong\langle 1,-a\rangle$.

\section{Corps quasi-pythagoriciens}

Le corps $F$ est dit quasi-pythagoricien lorsque $\mathrm{R}(F)=D_{F}(\langle 1,1\rangle)$. Cette notion a été introduite par Kijima et Nishi dans [10]. Nous allons donner différentes caractérisations des corps quasi-pythagoriciens, pour la plupart connues. Elles permettent de donner une nouvelle preuve, de nature structurelle, d'un résultat important de [10], à savoir qu'une extension quadratique $F(\sqrt{a})$ d'un corps quasi-pythagoricien $F$ est également un corps quasipythagoricien lorsque $a \in \sum F^{\times^{2}}(5.2)$. En ce qui concerne les corps quasipythagoriciens à ordre unique, il s'avère que ce sont exactement ceux décrits par I. Kaplansky dans le premier résultat sur le radical (5.3).

Une forme quadratique $\varphi$ est dite faiblement isotrope (resp. de torsion) s'il existe $n \geq 1$ tel que $n \times \varphi$ est isotrope (resp. hyperbolique). Une forme non faiblement isotrope est dite fortement anisotrope.

5.1 Proposition. Les conditions suivantes sont équivalentes :

(i) F est quasi-pythagoricien.

(ii) $\mathrm{R}(F)=\sum F^{\times^{2}}$.

(iii) Pour tout élément $a \in \sum F^{\times^{2}}$ on a l'égalité $D_{F}(\langle 1, a\rangle)=\mathrm{R}(F)$.

(iv) Il existe $a \in F^{\times}$tel que $D_{F}(\langle 1, a\rangle)=\mathrm{R}(F)$.

(v) $I^{2} F$ est sans torsion.

(vi) Sur F, toute 2-forme de Pfister anisotrope est fortement anisotrope.

(vii) L'homomorphisme $I^{2} F \rightarrow I^{3} F$ donné par la multiplication par la forme $\langle 1,1\rangle$ est injectif. 
Référence : Les équivalences $(i \Leftrightarrow i i \Leftrightarrow v)$ sont données dans [10, Section 2].

Démonstration : $(i \Rightarrow i i)$ : On suppose $D_{F}(\langle 1,1\rangle)=\mathrm{R}(F)$. Par $(2.1)$ et l'implication $(i \Rightarrow$ iii) de (3.1) on obtient

$$
D_{F}(\langle 1,1,1\rangle)=\bigcup_{x \in D_{F}(\langle 1,1\rangle)} D_{F}(\langle 1, x\rangle)=\bigcup_{x \in \mathrm{R}(F)} D_{F}(\langle 1, x\rangle)=D_{F}(\langle 1,1\rangle),
$$

i.e. dans $F$, tout élément qui est somme de trois carrés est déjà une somme de deux carrés. Il vient $\sum F^{\times^{2}}=D_{F}(\langle 1,1\rangle)=\mathrm{R}(F)$.

$(i i \Rightarrow$ iii $)$ : Pour $a \in \sum F^{\times^{2}}$, les inclusions $\mathrm{R}(F) \subset D_{F}(\langle 1, a\rangle) \subset \sum F^{\times^{2}}$ deviennent des égalités sous l'hypothèse $(i i)$.

$(i i i \Rightarrow i v):$ On prend $a:=1$.

(iv $\Rightarrow i)$ : Supposons que $D_{F}(\langle 1, a\rangle)=\mathrm{R}(F)$. Il vient $a \in \mathrm{R}(F)$ et par l'implication $(i \Rightarrow i i i)$ de $(3.1)$ il s'ensuit que $D_{F}(\langle 1,1\rangle)=D_{F}(\langle 1, a\rangle)=$ $\mathrm{R}(F)$.

$(i i \Rightarrow v)$ : Supposons que $\mathrm{R}(F)=\sum F^{\times^{2}}$. Soit $\varphi$ une forme anisotrope de dimension au moins trois sur $F$. On va montrer que $n \times \varphi$ est anisotrope pour tout $n \geq 1$. Soit $\varphi \cong\left\langle a_{1}, \ldots, a_{m}\right\rangle$ avec $a_{1}, \ldots, a_{m} \in F^{\times}$et $m \geq 3$. Si $n \times \varphi$ est isotrope pour un $n \geq 1$, alors il existe $r_{1}, \ldots, r_{m} \in \sum F^{\times 2}=\mathrm{R}(F)$ tels que $\left\langle r_{1} a_{1}, \ldots, r_{m} a_{m}\right\rangle$ est isotrope. C'est donc aussi vrai pour $\varphi$, d'après (4.2). Cela montre que toute forme anisotrope de dimension au moins 3 est fortement anisotrope. Il s'ensuit que $I^{2} F$ est sans torsion.

$(v \Rightarrow v i, v i i)$ : C'est clair.

$(v i \Rightarrow i i)$ : Soit $a \in \sum F^{\times^{2}}$. Pour tout $b \in F^{\times}$la forme $\langle\langle-a, b\rangle\rangle$ est de torsion, donc hyperbolique par l'hypothèse $(v i)$. Cela montre que $\langle 1,-a\rangle$ est universelle, d'où $a \in \mathrm{R}(F)$.

$(v i i \Rightarrow v)$ : Par hypothèse, l'idéal $I^{2} F$ ne contient pas d'élément d'ordre 2 . Or, l'ordre de tout élément de torsion de $W(F)$ est une puissance de 2 [18, 2.6.4. Theorem, (ii)]. Donc $I^{2} F$ est sans torsion.

5.2 Corollaire (Kijima, Nishi). Soient $F$ un corps quasi-pythagoricien et $K=F(\sqrt{a})$ pour $a \in \sum F^{\times 2}$. Alors $K$ est quasi-pythagoricien.

Référence: $[10$, p. 34].

Démonstration : La proposition (5.1) assure que $a \in \mathrm{R}(F)$ et que l'homomorphisme $I^{2} F \rightarrow I^{3} F$ donné par la multiplication par la forme $\langle 1,1\rangle$ est injectif. On peut supposer que $a \notin F^{\times^{2}}$. On considère le diagramme commutatif

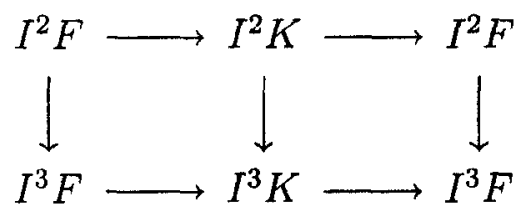


où les flèches verticales représentent les multiplications respectives avec la forme $\langle 1,1\rangle$ et où les flèches horizontales sont données par l'homomorphisme canonique $W(F) \rightarrow W(K)$ et le transfert $W(K) \rightarrow W(F)$ associé à la $F$-forme linéaire $s: K \rightarrow F$ avec $s(1)=0$ et $s(\sqrt{a})=1$ (cf. [18, Chap. 2, $\S 5])$. Il résulte du théorème (4.5) que l'homomorphisme $I^{3} F \rightarrow I^{3} K$ est injectif et de $[4$, Theorem 3.3.] que la première ligne du diagramme est exacte. Une chasse de diagramme montre alors que la flèche verticale du milieu est aussi injective. Ainsi, d'après (5.1), $K$ est quasi-pythagoricien.

\subsection{Corollaire (Kaplansky). Les conditions suivantes sont équivalentes:}

(i) F est réel et la seule $F$-algèbre de quaternions à division est $(-1,-1)_{F}$.

(ii) F est quasi-pythagoricien et admet un seul ordre.

(iii) $\mathrm{R}(F)$ est d'indice 2 dans $F^{\times}$.

Référence : L'équivalence $(i \Leftrightarrow i i i)$ est l'énoncé de [8, Theorem 1].

Démonstration : $(i \Rightarrow i i)$ : L'unique 2-forme de Pfister anisotrope sur $F$ est $\langle\langle 1,1\rangle\rangle$, la forme norme de $(-1,-1)_{F}$, et cette forme est fortement anisotrope car $F$ est réel. Par la caractérisation $(5.1$, vi) il suit que $F$ est quasi-pythagoricien. Pour $a \in F^{\times} \backslash D_{F}(\langle 1,1\rangle)$ la $F$-algèbre de quaternions $(-1, a)_{F}$ est à division, donc isomorphe à $(-1,-1)_{F}$ et il s'ensuit que $-a \in$ $D_{F}(\langle 1,1\rangle)$. Comme $F$ est réel, cela montre que $\sum F^{\times^{2}}$ est d'indice 2 dans $F^{\times}$, i.e. $F$ admet un seul ordre.

$\left(i i \Rightarrow\right.$ iii) : D'après les hypothèses, $\mathrm{R}(F)$ est égal à $\sum F^{\times 2}$ qui est d'indice 2 dans $F^{\times}$.

(iii $\Rightarrow i$ ) : On choisit $x \in F^{\times} \backslash \mathrm{R}(F)$. L'hypothèse et les relations $\mathrm{R}(F) \subset D_{F}(\langle 1,-x\rangle) \subsetneq F^{\times}$entrainent que $D_{F}(\langle 1,-x\rangle)=\mathrm{R}(F)$. Il s'ensuit par l'implication $(i v \Rightarrow i i)$ de la proposition (5.1) que $\mathrm{R}(F)=\sum F^{\times^{2}}$. Donc $F$ est réel, $F^{\times}=\mathrm{R}(F) \cup-\mathrm{R}(F)$ et la $F$-algèbre $(-1,-1)_{F}$ est à division.

Supposons maintenant que $(a, b)_{F}$ est à division, avec $a, b \in F^{\times}$. Il vient $a, b \notin \mathrm{R}(F)$, donc $-a,-b \in \mathrm{R}(F)$. A l'aide de la partie (c) de la proposition (4.2), on obtient $\langle\langle-a,-b\rangle\rangle \cong\left\langle\langle 1,1\rangle\right.$ et alors $(a, b)_{F} \cong(-1,-1)_{F}$.

Le corps des réels $\mathbb{R}$ satisfait les conditions équivalentes du corollaire (5.3). En effet, le théorème de Frobenius affirme que $(-1,-1)_{\mathbb{R}}$ est la seule $\mathbb{R}$-algèbre centrale à division. Pour tout entier positif $m, \mathrm{~K}$. Szymiczek a donné un exemple de corps réel $F$ tel que $\left|F^{\times} / F^{\times 2}\right|=2^{m}$ et tel que $\mathrm{R}(F)$ est d'indice 2 dans $F^{\times}[19$, p. 217]. On pourra également obtenir des exemples de tels corps en appliquant le corollaire (8.5) au corps $\mathbb{R}$. 


\section{Radical et extension du corps}

Cette section vise à établir des relations entre les deux radicaux $\mathrm{R}(F)$ et $\mathrm{R}(K)$ pour une extension $K / F$. D'abord, nous cherchons à savoir à quelle condition l'inclusion $\mathrm{R}(F) \subset \mathrm{R}(K)$ est valable. Ensuite, nous nous restreignons au cas d'une extension quadratique $K / F$ et nous abordons le problème de savoir si la suite exacte canonique qui fait intervenir les groupes des carrés

$$
1 \longrightarrow\left\{F^{\times 2}, a F^{\times 2}\right\} \longrightarrow F^{\times} / F^{\times 2} \longrightarrow K^{\times} / K^{\times^{2}} \longrightarrow F^{\times} / F^{\times 2}
$$

(voir proposition (2.4)) admet un analogue au moins partiel pour les radicaux. On obtient effectivement un complexe

$$
F^{\times} / \mathrm{R}(F) \longrightarrow K^{\times} / \mathrm{R}(K) \longrightarrow F^{\times} / \mathrm{R}(F) .
$$

La question de l'exactitude de ce complexe a été posée à l'origine par D. Kijima et M. Nishi. Nous nous inspirons là de l'étude entreprise dans [9], [10] et [7]. Enfin, nous donnons un exemple simple d'extension quadratique $K / F$ où $F$ est sans radical alors que $K$ est de radical non trivial.

6.1 Théorème (Cordes). Pour toute extension quadratique $K / F$ on a les inclusions $\mathrm{R}(F) \subset \mathrm{R}(K)$ et $N_{K / F}(\mathrm{R}(K)) \subset \mathrm{R}(F)$.

Référence : [3, Proposition 3, Corollary] et [3, Proposition 5].

Démonstration : Soit $a \in \mathrm{R}(F)$. Pour $x \in K^{\times}$, comme la forme $\langle 1,-a\rangle$ représente $N_{K / F}(x)$ sur $F$, le Principe de la Norme assure l'existence d'un élément $c$ de $F^{\times}$tel que $c x \in D_{K}(\langle 1,-a\rangle)$; comme $c \in D_{F}(\langle 1,-a\rangle)$, il vient $x \in D_{K}(\langle 1,-a\rangle)$. Ainsi la forme $\langle 1,-a\rangle$ est universelle aussi sur $K$. Cela montre l'inclusion $\mathrm{R}(F) \subset \mathrm{R}(K)$.

Pour voir que $N_{K / F}(\mathrm{R}(K)) \subset \mathrm{R}(F)$, considérons maintenant $x \in \mathrm{R}(K)$. Pour $a \in F^{\times}$la forme $\langle 1, a\rangle$ représente $x$ sur $K$; d'après le Principe de la Norme, la même forme sur $F$ représente alors $N_{K / F}(x)$. On en conlut que $N_{K / F}(x) \in \mathrm{R}(F)$.

6.2 Corollaire. Pour toute 2-extension $K / F$ on a $\mathrm{R}(F) \subset \mathrm{R}(K)$.

Démonstration : Soient $r \in \mathrm{R}(F)$ et $x \in K^{\times}$. Alors $F(x) / F$ est une 2-extension finie. À l'aide de (2.6) on choisit une suite de corps $F_{0}, \ldots, F_{n}$, où $F_{0}=F$ et $F_{n}=F(x)$, telle que $F_{i} / F_{i-1}$ est une extension quadratique pour $1 \leq i \leq n$. Par récurrence sur $i$, on obtient, grâce à (6.1), que $r \in \mathrm{R}\left(F_{i}\right)$. En particulier, $\langle 1,-r\rangle$ représente $x$ sur le corps $F_{n}=F(x)$, donc aussi sur $K$. Cela montre que $\mathrm{R}(F) \subset \mathrm{R}(K)$.

Pour une extension $K / F$ transcendante ou finie de degré autre que 2, il peut arriver que $\mathrm{R}(F) \not \subset \mathrm{R}(K)$. 
6.3 Exemples. (1) Soit $F$ un corps non quadratiquement clos tel que $\mathrm{R}(F)=F^{\times}$. Alors pour $K:=F((X))$ on a $\mathrm{R}(K)=K^{\times^{2}}(3.6)$ et $F^{\times} \not \subset K^{\times^{2}}$, d'où $\mathrm{R}(F) \not \subset \mathrm{R}(K)$.

(2) Soit $n$ un entier supérieur ou égal à 3 . Notons $F_{0}$ la clôture quadratique du corps $\mathbb{Q}$. On choisit une extension $K_{0} / F_{0}$ de degré $n$ (voir la remarque suivante). Il est connu que le groupe des classes de carrés de $K_{0}$ est infini [13, Chapter VII, Appendix, Corollary 3]. Posons $F:=F_{0}((X))$ et $K:=K_{0}((X))$. Il vient alors $[K: F]=\left[K_{0}: F_{0}\right]=n, \mathrm{R}(F)=F^{\times}$et $\mathrm{R}(K)=K^{\times^{2}}$. En particulier, $X \in \mathrm{R}(F) \backslash \mathrm{R}(K)$.

Remarque: Pour obtenir une extension $K_{0} / F_{0}$ de degré $n \geq 3$ donné, où $F_{0}$ est la clôture quadratique de $\mathbb{Q}$, on peut utiliser la construction suivante, qui a été suggerée à l'auteur par D. Leep :

On choisit une extension galoisienne $L / \mathbb{Q}$ de groupe de Galois $\mathcal{S}_{n}$ et on note $k$ le sous-corps de $L$ fixé par $\mathcal{A}_{n}$. Ainsi $k / \mathbb{Q}$ est une extension quadratique et $L / k$ une extension galoisienne de groupe $\mathcal{A}_{n}$. Comme $\mathcal{A}_{n}$ n'a pas de sous-groupe d'indice 2 , le corps $L$ ne contient aucune sous-extension quadratique de $k$. Ceci dit que les extensions $L / k$ et $F_{0} / k$ sont linéairement disjointes. Ainsi $L F_{0} / F_{0}$ est également une extension galoisienne de groupe $\mathcal{A}_{n}$. On choisit un sous-groupe $H$ de $\mathcal{A}_{n}$ isomorphe à $\mathcal{A}_{n-1}$, donc d'indice $n$. Alors le sous-corps de $L F_{0}$ fixé par $H$ est une extension de degré $n$ de $F_{0}$.

6.4 Corollaire. Pour toute extension quadratique $K / F$, les homomorphismes d'inclusion $F^{\times} \hookrightarrow K^{\times}$et de norme $N_{K / F}: K^{\times} \rightarrow F^{\times}$induisent un complexe

$$
F^{\times} / \mathrm{R}(F) \longrightarrow K^{\times} / \mathrm{R}(K) \longrightarrow F^{\times} / \mathrm{R}(F) .
$$

Démonstration : Le fait que les deux homomorphismes soient bien définis provient du théorème. Les relations $N_{K / F}\left(F^{\times}\right)=F^{\times^{2}} \subset \mathrm{R}(F)$ montrent alors que leur composition dans l'ordre donné est triviale.

6.5 Corollaire. Soit $K / F$ une extension quadratique telle que $K=F(\sqrt{a})$ pour $a \in \mathrm{R}(F) \backslash F^{\times^{2}}$. Alors on a l'égalité $\mathrm{R}(F)=F^{\times} \cap \mathrm{R}(K)$ et le complexe

$$
1 \longrightarrow F^{\times} / \mathrm{R}(F) \longrightarrow K^{\times} / \mathrm{R}(K) \longrightarrow F^{\times} / \mathrm{R}(F) \longrightarrow 1
$$

issu de (6.4) est exact à gauche et à droite.

Démonstration : Soit $x \in F^{\times} \cap \mathrm{R}(K)$. Pour $y \in F^{\times}$, la $F$-algèbre de quaternions $(x, y)_{F}$, devenant déployée après extension des scalaires à $K$, est elle-même déjà déployée, puisque $\operatorname{Br}(K / F)=0$ (3.1). Par conséquent, $x \in \mathrm{R}(F)$. Cela montre qu'ici l'inclusion $\mathrm{R}(F) \subset F^{\times} \cap \mathrm{R}(K)$ (6.1) est une 
égalité. Aussi le complexe est-il exact à gauche. Comme $a \in \mathrm{R}(F)$ la norme $N_{K / F}$ est surjective, d'où l'exactitude à droite.

La question de l'exactitude du complexe du corollaire (6.4) est équivalente à :

6.6 Question (Kijima, Nishi). Pour $K / F$ une extension quadratique, a-t-on l'égalité $N_{K / F}^{-1}(\mathrm{R}(F))=F^{\times} \mathrm{R}(K)$ ?

David Leep a annoncé récemment un exemple qui montre que la réponse à cette question, en général, est négative : il construit une extension quadratique $K / F$ telle que $\mathrm{R}(F) \neq F^{\times^{2}}$ alors que $\mathrm{R}(K) \subset F^{\times} K^{\times^{2}}$; il s'ensuit que $F^{\times} \mathrm{R}(K)=N_{K / F}^{-1}\left(F^{\times 2}\right) \neq N_{K / F}^{-1}(\mathrm{R}(F))$, puisque $\mathrm{R}(F) \subset N_{K / F}\left(K^{\times}\right)$.

Néanmoins, dans certains cas, la question admet une réponse positive :

6.7 Corollaire (Kijima, Nishi). Soient $F$ un corps quasi-pythagoricien et $K=F(\sqrt{a})$ avec $a \in \mathrm{R}(F) \backslash F^{\times^{2}}$. Alors le complexe donné dans (6.5) est exact.

Référence : [10, Theorem 2.13.].

Démonstration : Comme $F$ est quasi-pythagoricien, d'après (5.2), il en est de même pour $K$. À l'aide du Principe de la Norme, on obtient

$$
N_{K / F}^{-1}(\mathrm{R}(F))=N_{K / F}^{-1}\left(D_{F}(\langle 1,1\rangle)\right)=F^{\times} D_{K}(\langle 1,1\rangle)=F^{\times} \mathrm{R}(K),
$$

ce qui prouve l'exactitude du complexe au milieu.

Pour une extension quadratique $K=F(\sqrt{a})$, le corollaire (6.5) affirme que l'inclusion $\mathrm{R}(F) \subset F^{\times} \cap \mathrm{R}(K)$ est une égalité lorsque $a \in \mathrm{R}(F)$. Pourtant, si $a \notin \mathrm{R}(F)$, on peut avoir une inclusion stricte $\mathrm{R}(F) \subseteq F^{\times} \cap \mathrm{R}(K)$ comme l'illustre l'exemple suivant.

6.8 Exemple. Soient $F$ un sous-corps maximal réel de $\mathbb{Q}_{2}$ et $K=F(\sqrt{-2})$. Alors $F$ est sans radical, tandis que $\mathrm{R}(K)$ contient une classe non triviale modulo $K^{\times^{2}}$ représentée par -7 . En effet, avec $a:=2, b:=5$ et $c:=7$, on vérifie que les hypothèses de (6.9) ci-dessous sont satisfaites, en utilisant que $F(\sqrt{-7})$ est l'unique extension quadratique de $F$ contenue dans $\mathbb{Q}_{2}$ et que 7 n'est une somme de trois carrés ni dans $F$ ni dans $\mathbb{Q}_{2}$.

Par ailleurs, dans [1, Section 2], L. Berman construit, pour tout $n \geq 0$, un corps réel pythagoricien $F$ tel que le corps $K:=F(\sqrt{-1})$ vérifie les conditions $\left|K^{\times} / \mathrm{R}(K)\right|=4$ et $\left|\mathrm{R}(K) / K^{\times 2}\right|=2^{n}$; pour l'extension quadratique $K / F$ on a alors $\mathrm{R}(F) \subsetneq F^{\times} \cap \mathrm{R}(K)$ si $n>0$. 
6.9 Proposition. Soient $F$ un corps réel tel que $\left|F^{\times} / F^{\times^{2}}\right|=16$ et $a, b$ des éléments de $D_{F}(\langle 1,1\rangle)$ tels que $a+b$ n'est pas somme de 3 carrés dans $F$. On note $K:=F(\sqrt{-a})$ et $c:=a+b$. Alors :

(a) F est sans radical et admet un unique ordre,

(b) $D_{F}(\langle 1, c\rangle)=\sum F^{x^{2}}$,

(c) $K$ est non réel avec $\left|K^{\times} / K^{\times^{2}}\right|=32$,

(d) $\mathrm{R}(K)=K^{\times^{2}} \cup-c K^{\times^{2}}$ et $-c \notin K^{\times^{2}}$.

Démonstration : Pour $a_{1}, \ldots, a_{m} \in F^{\times}$on note $\operatorname{gr}\left(a_{1}, \ldots, a_{m}\right)$ le sousgroupe de $F^{\times}$engendré par $a_{1}, \ldots, a_{m}$.

(a) On montre d'abord que les éléments $a$ et $b$ représentent des classes distinctes et non triviales modulo le radical de $F$. Si l'on avait $a \in \mathrm{R}(F)$ alors on obtiendrait par $\left(4.2\right.$, a) que $D_{F}(\langle a, b\rangle)=D_{F}(\langle 1, b\rangle) \subset D_{F}(\langle 1,1,1\rangle)$, ce qui est impossible car $a+b$ n'est pas somme de 3 carrés dans $F$. On conclut que $a \notin \mathrm{R}(F)$ et, par le même argument, que $b \notin \mathrm{R}(F)$. Enfin, si l'on suppose que $a b \in \mathrm{R}(F)$, alors on obtient $D_{F}(\langle 1, a b\rangle)=D_{F}(\langle 1,1\rangle) \ni a$ et par suite $a+b=a\left(1+\left(a^{-1}\right)^{2} a b\right) \in D_{F}(\langle 1,1\rangle)$, ce qui est aussi une contradiction avec l'hypothèse que $a+b$ n'est pas somme de moins de 4 carrés dans $F$.

Il s'ensuit que $\mathrm{R}(F)$ est d'indice 4 dans $g r(a, b) \mathrm{R}(F)$. Étant donné que $\left|F^{\times} / F^{\times 2}\right|=16$, on déduit de la chaine d'inclusions

$$
F^{x^{2}} \subset \mathrm{R}(F) \subset g r(a, b) \mathrm{R}(F) \subset D_{F}(\langle 1,1\rangle) \subsetneq \sum F^{x^{2}} \subsetneq F^{\times}
$$

les égalités $\mathrm{R}(F)=F^{\times 2}$ et $D_{F}(\langle 1,1\rangle)=g r(a, b) F^{\times^{2}}$, ainsi que le fait que $\sum F^{\times^{2}}$ soit d'indice 2 dans $F^{\times}$, ce qui est équivalent à ce que le seul ordre de $F$ soit $\sum F^{\times^{2}}$.

(b) En plus, on obtient $\sum F^{\times^{2}}=g r(a, b, c) F^{\times^{2}}$ car $c \notin D_{F}(\langle 1,1\rangle)$. Il est clair que $D_{F}(\langle 1, a\rangle) \subset D_{F}(\langle 1,1,1\rangle) \not \nexists c$ et que $D_{F}(\langle 1, a\rangle) \not \subset D_{F}(\langle 1,1\rangle)$. Il vient $D_{F}(\langle 1, a\rangle) \cap D_{F}(\langle 1,1\rangle)=F^{\times^{2}} \cup a F^{\times^{2}}$, puis $D_{F}(\langle 1, a\rangle)=\operatorname{gr}(a, b c) F^{\times^{2}}$. Par conséquent, la $F$-algèbre de quaternions $(-c, a)_{F}$, isomorphe à $(-b, a c)_{F}$ puisque $-c+a=-b$, est déployée. Par symétrie des hypothèses sur $a$ et $b$, on a également que $(-c, b)_{F}$ est déployée. Cela montre que $a, b, c \in D_{F}(\langle 1, c\rangle)$, d'où $D_{F}(\langle 1, c\rangle)=\sum F^{\times^{2}}$.

(c) Il est clair que $K$ est non réel. L'image de la norme $N_{K / F}: K^{\times} \rightarrow F^{\times}$ est $D_{F}(\langle 1, a\rangle)=\operatorname{gr}(a, b c) F^{\times^{2}}$. Par (2.4) on en déduit que $\left|K^{\times} / K^{\times 2}\right|=$ $\frac{1}{2} \cdot\left|F^{\times} / F^{\times 2}\right| \cdot\left|D_{F}(\langle 1, a\rangle) / F^{\times 2}\right|=32$.

(d) L'élément $-c$ n'est pas un carré dans $K$. Comme $\sum F^{\times^{2}}=D_{F}(\langle 1, c\rangle)$ est un ordre de $F$ et $-a \in-\sum F^{\times^{2}} \cap K^{\times 2}$, il vient $F^{\times} \subset D_{K}(\langle 1, c\rangle)$. D'un 
autre côté, $N_{K / F}\left(K^{\times}\right)=D_{F}(\langle 1, a\rangle) \subset \sum F^{\times^{2}}=D_{F}(\langle 1, c\rangle)$. Ainsi le principe de la Norme assure que la forme $\langle 1, c\rangle$ est universelle sur $K$, i.e. que $-c \in$ $\mathrm{R}(K)$. Cela montre que $K^{\times^{2}} \cup-c K^{\times^{2}} \subset \mathrm{R}(K)$.

Montrons l'inclusion réciproque. Considérons un élément $x \in \mathrm{R}(K)$. Par le théorème (6.1) et par (a), on obtient $N_{K / F}(x) \in \mathrm{R}(F)=F^{\times^{2}}$. En vertu de (2.4), on peut alors supposer que $x \in F^{\times}$. D'après le Principe de la Norme, la forme $\langle 1,-x\rangle$, qui est universelle sur $K$, représente sur $F$ toutes les normes de $K / F$, et donc, en particulier, les éléments $a$ et $b c$. Ainsi $x \in$ $D_{F}(\langle 1,-a\rangle) \cap D_{F}(\langle 1,-b c\rangle)$. Comme $\mathrm{R}(F)=F^{\times^{2}}$ et $\left|F^{\times} / F^{x^{2}}\right|=16$, chacune des formes $\langle 1,-a\rangle$ et $\langle 1,-b c\rangle$ représente sur $F$ au plus 8 classes de carrés. De $-1,-a,-c \in D_{F}(\langle 1,-a\rangle)$ et de $-a,-c,-b c \in D_{F}(\langle 1,-b c\rangle)$ on déduit que $D_{F}(\langle 1,-a\rangle)=\operatorname{gr}(-1, a, c) F^{\times^{2}}$ et $D_{F}(\langle 1,-b c\rangle)=\operatorname{gr}(-a, b,-c) F^{\times^{2}}$. Il vient alors $x \in \operatorname{gr}(-a,-c) F^{\times^{2}} \subset K^{\times 2} \cup-c K^{\times 2}$.

\section{Un problème de réduction d'indice}

Considérons ici une extension quadratique $K / F$ dont la forme norme $N_{K / F}$ est surjective. On peut alors écrire $K=F(\sqrt{a})$ avec $a \in \mathrm{R}(F) \backslash F^{\times^{2}}$ et on sait que l'homomorphisme canonique $\operatorname{Br}(F) \rightarrow \operatorname{Br}(K)$ est injectif (3.1). On connaît également le noyau de l'homomorphsime canonique $W(F) \rightarrow W(K)$ : il contient une seule classe non triviale représentée par la forme $\langle 1,-a\rangle$. En fait, il s'est avéré qu'aucune forme anisotrope sur $F$ autre que $\langle 1,-a\rangle$ ne peut devenir isotrope sur $K(4.5)$. On se demande alors si l'on ne peut pas constater un fait plus fort que l'injectivité de $\operatorname{Br}(F) \rightarrow \operatorname{Br}(K)$, qui serait l'absence de réduction de l'indice pour toute $F$-algèbre simple centrale lors de l'extension des scalaires à $K$. On formule ce problème de la manière suivante :

7.1 Question. Soit $K / F$ une extension quadratique comme ci-dessus. Existe-t-il une F-algèbre centrale à division $D$ telle que $D_{K}$ ne soit pas à division?

Remarques : (1) Pour répondre à cette question, on peut se restreindre à considérer les $F$-algèbres centrales à division d'exposant une puissance de 2 . En effet, le théorème de décomposition primaire pour les algèbres simples centrales (cf. [15, p. 261] ou [16, Theorem 7.2.13]) assure que $D \cong D_{1} \otimes_{F} D_{2}$ pour deux $F$-algèbres simples centrales $D_{1}$ et $D_{2}$ telles que $D_{1}$ est de degré impair et $D_{2}$ est de degré une puissance de 2 ; ainsi $\left(D_{1}\right)_{K}$ est à division puisque $[K: F]=2$ et par suite $D_{K}$ est à division si et seulement si $\left(D_{2}\right)_{K}$ est à division $[15, \S 14.4$, Proposition b (viii)]. 
(2) Pour une extension $K / F$ quelconque, il peut arriver que $\operatorname{Br}(K / F)=0$ alors qu'il existe une $F$-algèbre centrale à division sur $F$ qui ne reste pas à division après extension des scalaires à $K$. C'est le cas dans l'exemple cidessous. Pour autant, on ne connait pas d'exemple d'une telle situation où l'extension soit finie [17, p. 22, Remark].

7.2 Exemple. Soit $B$ une $F$-algèbre de biquaternions à division. Notons $\varphi$ sa forme d'Albert, définie à similitude près, et $K$ le corps de fonctions $F(\varphi)$. Alors l'homomorphisme $\mathrm{Br}(F) \rightarrow \operatorname{Br}(K)$ est injectif et $B_{K}$ n'est pas à division. Ces deux constatations se font, par exemple, à partir de la formule, due à $\mathrm{A}$. Merkurjev, de réduction d'indice sur le corps de fonctions d'une quadrique [20].

7.3 Proposition. Soient $A$ une $F$-algèbre simple centrale et $K=F(\sqrt{a})$ avec $a \in \mathrm{R}(F)$. Alors $\exp _{K}\left(A_{K}\right)=\exp _{F}(A)$. De plus, si $\operatorname{ind}_{F}(A)=\exp _{F}(A)$ alors $\operatorname{ind}_{K}\left(A_{K}\right)=\operatorname{ind}_{F}(A)$.

Démonstration: L'ordre de $[A]$ dans $\operatorname{Br}(F)$ et de $\left[A_{K}\right]$ dans $\operatorname{Br}(K)$ est le même parce que l'homomorphisme canonique $\operatorname{Br}(F) \rightarrow \operatorname{Br}(K)$ est injectif. Cela dit que $\exp _{K}\left(A_{K}\right)=\exp _{F}(A)$. Sous l'hypothèse supplémentaire $\operatorname{ind}_{F}(A)=\exp _{F}(A)$, les inégalités $\exp _{K}\left(A_{K}\right) \leq \operatorname{ind}_{K}\left(A_{K}\right) \leq \operatorname{ind}_{F}(A)$ (valables pour toute extension $K / F$ ) sont alors des égalités.

7.4 Corollaire. Soient $F$ une extension algébrique de $\mathbb{Q}$ et $K=F(\sqrt{a})$ avec $a \in \mathrm{R}(F)$. Si $D$ est une $F$-algèbre centrale à division alors $D_{K}$ est aussi à division.

Démonstration : L'égalité $\operatorname{ind}_{F}(A)=\exp _{F}(A)$ vaut pour toute $F$-algèbre simple centrale $A$ sur un corps de nombre $F$, par conséquent aussi, lorsque $F$ est une extension algébrique de $\mathbb{Q}$. À l'aide de (7.3) on conclut aux égalités $\operatorname{ind}_{K}\left(D_{K}\right)=\operatorname{ind}_{F}(D)=\operatorname{deg}_{F}(D)=\operatorname{deg}_{K}\left(D_{K}\right)$ qui montrent que $D_{K}$ est à division.

Remarque: La conclusion est valable dès que $F$ est un corps tel que $\operatorname{ind}_{F}(A)=\exp _{F}(A)$ pour toute $F$-algèbre simple centrale $A$. Cette condition est satisfaite par d'autres types intéressants de corps que les extensions algébriques de $\mathbb{Q}$.

7.5 Proposition. Soit $K=F(\sqrt{a})$ avec $a \in \mathrm{R}(F)$. Soit $D$ une $F$-algèbre centrale $\dot{a}$ division de degré $2^{n}, n \geq 1$. Si toute extension $L / F$ contenue dans $D$ et de degré divisant $2^{n-1}$ est telle que $\mathrm{R}(F) \subset \mathrm{R}(L)$ alors $D_{K}$ est à division. 
Démonstration: Supposons que $D_{K}$ n'est pas à division. On sait que l'extension quadratique $K / F$ se plonge alors dans $D[15, \S 13.4$, Corollary]. Ainsi il existe $\alpha \in D$ tel que $\alpha^{2}=a$. En vertu du théorème de SkolemNoether, l'automorphisme d'extension non trivial de $F(\alpha) / F$ s'étend à $D$ en un automorphisme intérieur $\operatorname{Int}(u)$ avec $u \in D^{\times}$. Cet élément $u$ anticommute alors avec $\alpha$. Tous les éléments de $F\left(u^{2}\right)$ commutent avec $\alpha$ ce qui n'est pas le cas de $u$. Il vient $F\left(u^{2}\right) \subsetneq F(u)$, donc $L:=F\left(u^{2}\right)$ n'est pas un sous-corps maximal de $D$ et alors $[L: F]$ divise $2^{n-1}$. Les éléments $\alpha$ et $u$ engendrent une $L$-algèbre de quaternions $Q$, contenue dans $D$ et donc nécessairement à division. De plus, $Q$ est déployée par l'extension quadratique $L(\sqrt{a})$. Ainsi $\operatorname{Br}(L(\sqrt{a}) / L)$ n'est pas trivial et par suite $a \notin \mathrm{R}(L)$. Donc $\mathrm{R}(F) \not \subset \mathrm{R}(L)$, contrairement à l'hypothèse.

7.6 Corollaire. Soient $K=F(\sqrt{a})$ avec $a \in \mathrm{R}(F)$ et $D$ une $F$-algèbre centrale à division telle que $D_{K}$ n'est pas à division. Alors le degré de $D$ est divisible par 8 et strictement supérieur à l'exposant de D.

Démonstration: En vertu de la remarque qui suit la question (7.1), on peut supposer que $D$ est de degré $2^{n}$, avec $n \geq 1$. Comme ind ${ }_{K}\left(D_{K}\right)<\operatorname{ind}_{F}(D)$ par hypothèse, (7.3) montre que $\exp _{F}(D)<\operatorname{ind}_{F}(D)$. D'après $(7.5), D$ contient une extension $L$ de $F$ telle que $[L: F]$ divise $2^{n-1}$ et $\mathrm{R}(F) \not \subset \mathrm{R}(L)$. D'après (6.1), $L / F$ n'est pas une extension quadratique, d'où $n \geq 3$, ce qui achève la preuve.

Remarque: Sous les hypothèses du corollaire, si le degré de $D$ est 8 alors son exposant est 4 . En effet, le corollaire dit que $\exp _{F}(D) \leq 4$ et nous allons voir que $\exp _{F}(D) \neq 2$. Si $\exp _{F}(D)=2$ alors un théorème de Rowen et sa preuve [16, p. 279-280, Exercise 32] montrent qu'il existe $b, c \in F^{\times}$tels que $D$ est déployée par $F(\sqrt{a}, \sqrt{b}, \sqrt{c})$; pour $L:=F(\sqrt{b}, \sqrt{c})$ le corollaire (6.2) dit que $a \in \mathrm{R}(L)$, ce qui est en contradiction avec le fait que $D_{L}$ représente une classe non triviale dans $\operatorname{Br}(L(\sqrt{a}) / L)$.

\section{Agrandissement et réduction du radical}

Nous avons vu que le radical $\mathrm{R}(F)$ se projette de façon naturelle sur un idéal de l'anneau de Witt $W(F)(4.4)$. Notons $K W(F)$ l'anneau quotient de $W(F)$ par cet idéal. Nous nous intéressons aux extensions $K / F$ pour lesquelles les anneaux $K W(F)$ et $K W(K)$ sont canoniquement isomorphes. En quelque sorte, on pourrait dire que les corps $F$ et $K$ présentent alors au radical près la même structure pour les formes quadratiques. 
En premier lieu, nous construisons une extension $K$ d'un corps donné $F$ telle que toute forme quadratique anisotrope sur $F$ reste anisotrope sur $K$ et telle que $K^{\times}=F^{\times} \mathrm{R}(K)$ et $\mathrm{R}(F) K^{\times 2} \subsetneq \mathrm{R}(K)((8.4)$ et (8.5)). On parvient ainsi à agrandir le radical du corps en conservant sa structure pour les formes quadratiques au radical près. Cette approche conduit en particulier à de nouveaux exemples de corps $K$ pour lesquels le quotient $\mathrm{R}(K) / K^{\times 2}$ est fini de cardinal arbitraire.

Ensuite, nous étudions le problème inverse, qui consiste à réduire par extension le radical du corps. Pour tout corps $F$, nous établissons l'existence d'une plus petite 2-extension $K$ de $F$ sans radical (8.9). Sa construction rappelle celle de la clôture pythagoricienne d'un corps, avec des propriétés comparables (voir $[18, \S 2.6]$ ).

Un élément $x \in F^{\times} \backslash F^{\times 2}$ est dit rigide (dans $\left.F\right)$ si $D_{F}(\langle 1, x\rangle)=F^{\times 2} \cup x F^{\times 2}$. On dit que $x$ est birigide si $x$ et $-x$ sont rigides.

8.1 Proposition. Soient a un élément birigide dans $F, K:=F(\sqrt{a})$ et $\varphi$ une forme quadratique sur $F$.

(a) $K^{\times}=\left(F^{\times} \cup \sqrt{a} F^{\times}\right) K^{\times 2}$.

(b) Dans $K$ les deux racines carrées de a sont birigides.

(c) Si $\varphi_{K}$ est anisotrope alors $D_{K}\left(\varphi_{K}\right) \subset F^{\times} K^{\times 2}$.

(d) Pour que $\varphi_{K}$ soit anisotrope il faut et il suffit que $\varphi \perp$ a $\varphi$ soit anisotrope.

Démonstration: (a) Comme $-a$ est rigide on a $N_{K / F}\left(K^{\times}\right)=D_{F}(\langle 1,-a\rangle)=$ $F^{\times 2} U-a F^{\times 2}$. On en déduit l'énoncé à l'aide des égalités $N_{K / F}(\sqrt{a})=-a$ et $N_{K / F}^{-1}\left(F^{\times 2}\right)=F^{\times} K^{\times 2}(2.4)$.

(b) Soit $\xi$ l'une des deux racines carrées de $a$ dans $K$. Pour $b \in F^{\times}$tel que $b \notin F^{\times 2} \cup a F^{\times 2}=D_{F}(\langle 1, a\rangle)$ on a $N_{K / F}(-\xi)=-a \notin D_{F}(\langle 1,-b\rangle)$; alors, d'après le Principe de la Norme, $-\xi \notin D_{K}(\langle 1,-b\rangle)$, i.e. $b \notin D_{K}(\langle 1, \xi\rangle)$. Cela montre que $F^{\times} \cap D_{K}(\langle 1, \xi\rangle) \subset F^{\times^{2}} \cup a F^{\times^{2}} \subset K^{\times^{2}}$. Par $(a)$ on conclut que $D_{K}(\langle 1, \xi\rangle)=K^{\times 2} \cup \xi K^{\times 2}$. Ainsi $\xi$ est rigide dans $K$. Le même raisonnement vaut pour $-\xi$.

(c) Supposons que $\varphi_{K}$ est anisotrope et de dimension $n$. Par récurrence sur $n$ nous allons montrer que $D_{F}(\varphi) \subset F^{\times} K^{\times 2}$. Pour $n=1$ c'est clair. Dans le cas $n=2$ nous avons $\varphi \cong b\langle 1,-c\rangle$ avec $b, c \in F^{\times}$et $c \notin F^{\times 2} \cup a F^{\times 2}$. Supposons que $\varphi_{K}$ représente $\zeta \in K^{\times}$. Alors $b \zeta \in D_{K}(\langle 1,-c\rangle)$ entraîne par le Principe de la Norme que $N_{K / F}(\zeta) \in D_{F}(\langle 1,-c\rangle) \cap D_{F}(\langle 1,-a\rangle)$. Du fait que $a$ soit birigide et que $c \notin F^{\times 2} \cup a F^{\times 2}$ on déduit que $D_{F}(\langle 1,-c\rangle) \cap D_{F}(\langle 1,-a\rangle)$ 
est égal à $F^{\times^{2}}$. Ainsi $N_{K / F}(\zeta) \in F^{\times^{2}}$, ce qui, d'après (2.4), est équivalent à ce que $\zeta \in F^{\times} K^{\times^{2}}$.

Si $n>2$ nous écrivons $\varphi \cong \varphi^{\prime} \perp\langle c\rangle$ avec $\varphi^{\prime}$ une forme de dimension $n-1$ sur $F$ et $c \in F^{\times}$. On appliquant l'hypothèse de récurrence à $\varphi^{\prime}$ on obtient $D_{K}\left(\varphi_{K}^{\prime}\right) \subset F^{\times} K^{\times 2} \backslash-c K^{\times 2}$. Le lemme (2.1) et le cas $n=2$ permettent de conclure que

$$
D_{K}(\varphi)=\bigcup_{b \in D_{K}\left(\varphi_{K}^{\prime}\right)} D_{K}(\langle b, c\rangle)=\bigcup_{b \in D_{F}\left(\varphi^{\prime}\right)} D_{K}(\langle b, c\rangle) \subset F^{\times} K^{\times 2} .
$$

(d) On peut supposer que $\varphi$ est anisotrope. Si $\varphi \perp a \varphi$ est isotrope alors il existe une sous-forme binaire $\beta$ de $\varphi$ telle que $\beta \perp a \beta$ est isotrope $[5$, Proposition 2.2.], donc telle que $d_{ \pm}(\beta) \in D_{F}(\langle 1, a\rangle)=F^{\times^{2}} \cup a F^{\times^{2}} \subset K^{\times^{2}}$, ce qui entraîne que $\beta_{K}$ et $\varphi_{K}$ sont isotropes. Réciproquement, si $\varphi_{K}$ est isotrope alors $\varphi$ contient une sous-forme semblable à $\langle 1,-a\rangle[13$, Chap. VII, Lemma 3.2.] ; il s'ensuit que $\varphi \perp$ a $\varphi$ est isotrope.

Soit $a \in F$. Il existe un corps engendré par une suite d'éléments $\left(\alpha_{i}\right)_{i \geq 0}$ vérifiant $\alpha_{0}=a$ et $\alpha_{i}^{2}=\alpha_{i-1}$ pour $i \geq 1$. Un tel corps est unique à $F$-isomorphisme près. On le note $F(\sqrt[\infty]{a})$.

8.2 Proposition. Soient a un élément birigide dans $F$ et $\varphi$ une forme quadratique sur $F$. On pose $L:=F(\sqrt[\infty]{a})$.

(a) On a les égalités $L^{\times}=F^{\times} L^{\times^{2}}$ et $F^{\times} \cap L^{\times 2}=F^{\times 2} \cup a F^{\times^{2}}$.

(b) Pour que $\varphi_{L}$ soit anisotrope il faut et il suffit que $\varphi \perp$ a $\varphi$ soit anisotrope.

Démonstration: Soit $\left(\alpha_{i}\right)_{i \geq 0}$ une suite d'éléments de $L$ comme ci-dessus. On note $F_{i}:=F\left(\alpha_{i}\right)$ pour $i \geq 0$. Alors $L / F$ est la limite directe des extensions $F_{i} / F$. À l'aide de la proposition précédente on obtient par récurrence sur $i$ que $\alpha_{i}$ est un élément birigide dans $F_{i}$ et que

$$
F_{i}^{\times}=\left(F_{i-1}^{\times} \cup \alpha_{i} F_{i-1}^{\times}\right) F_{i}^{\times 2} \subset F_{i-1}^{\times} F_{i+1}^{\times}
$$

pour tout $i \geq 1$. Il découle de ces inclusions que $L^{\times}=F^{\times} L^{\times 2}$.

Si $\varphi_{L}$ est anisotrope alors $\varphi_{F_{1}}$ est anisotrope ; par la partie $(8.1, \mathrm{~d})$, il vient que $\varphi \perp a \varphi$ est anisotrope sur $F$. Pour la réciproque, supposons que $\varphi \perp a \varphi$ est anisotrope. Alors les parties (c) et (d) de (8.1) montrent que $\varphi_{F_{1}}$ est anisotrope et que $D_{F_{1}}\left(\varphi_{F_{1}}\right) \subset F^{\times} F_{1}^{\times 2}$. D'un autre côté, $N_{F_{1} / F}\left(\alpha_{1}\right)=$ $-a \notin F^{\times^{2}}$ implique que $\alpha_{1} \notin F^{\times} F_{1}^{x^{2}}$. Il s'ensuit que $\varphi_{F_{1}} \perp \alpha_{1} \varphi_{F_{1}}$ est anisotrope. On montre alors par récurrence que $\varphi_{F_{i}} \perp \alpha_{i} \varphi_{F_{i}}$ est anisotrope pour tout $i$. Par conséquent, $\varphi_{L}$ est anisotrope. 
En particulier, pour $x \in F^{\times}$la forme $\langle 1,-x\rangle$ devient hyperbolique sur $L$ si et seulement si $\langle\langle-x, a\rangle\rangle$ est hyperbolique sur $F$. On en déduit que $F^{\times} \cap L^{\times 2}=D_{F}(\langle 1, a\rangle)=F^{\times^{2}} \cup a F^{\times^{2}}$.

Désignons par $F\langle X\rangle$ la 2-extension maximale du corps des fonctions $F(X)$ dans le corps des séries formelles $F((X))$. La théorie de base des formes quadratiques sur un corps complet pour une valuation non 2 -adique $[13$, Chap. VI, Section 1] assure que $F((X))^{\times}=\left(F^{\times} \cup X F^{\times}\right) F((X))^{x^{2}}$ et que l'élément $X$ est birigide dans $F((X))$. Par définition de $F\langle X\rangle$, il vient que $X$ est aussi birigide dans $F\langle X\rangle$ ainsi que $F\langle X\rangle^{\times}=\left(F^{\times} \cup X F^{\times}\right) F\langle X\rangle^{\times 2}$.

8.3 Corollaire. Soit $L$ l'un des corps $F((X))(\sqrt[\infty]{X})$ ou $F\langle X\rangle(\sqrt[\infty]{X})$. Alors $L^{\times}=F^{\times} L^{\times^{2}}$ et toute forme quadratique anisotrope sur $F$ reste anisotrope après extension des scalaires $\dot{a} L$.

Référence : Cet énoncé est une variante de [11, Lemma 3.2.].

Démonstration : Écrivons $L=K(\sqrt[\infty]{X})$ où $K$ est l'un des corps $F((X))$ ou $F\langle X\rangle$. L'élément $X$ est birigide dans $K$ et devient un carré dans $L$. Comme $K^{\times}=\left(F^{\times} \cup X F^{\times}\right) K^{\times 2}$, on déduit de la proposition que $L^{\times}=F^{\times} L^{\times 2}$. Soit $\varphi$ une forme quadratique anisotrope sur $F$. Alors $\varphi_{K} \perp X \varphi_{K}$ est anisotrope [13, Chap. VI, Proposition 1.19.] ce qui implique, d'après la proposition, que $\varphi_{L}$ est anisotrope.

8.4 Théorème. Pour tout corps $F$ de caractéristique différente de 2 il existe une extension $K / F$ de degré de transcendance 1 telle que toute forme anisotrope sur $F$ reste anisotrope après extension des scalaires à $K$ et telle que les égalités $K^{\times}=\left(F^{\times} \cup r F^{\times}\right) K^{x^{2}}$ et $\mathrm{R}(K)=(\mathrm{R}(F) \cup r \mathrm{R}(F)) K^{\times^{2}}$ sont vérifiées pour un certain élément $r \in \mathrm{R}(K) \backslash F^{\times} K^{\times 2}$.

Démonstration : Posons $I:=F^{\times} / F^{x^{2}}$. Soit $\left(b_{\alpha}\right)_{\alpha \in I} \subset F^{\times}$un système complet de représentants des classes de carrés de $F$, où $b_{\alpha} \in \alpha$ pour tout $\alpha \in I$. Comme les $b_{\alpha}$ sont nécessairement distincts deux à deux, la famille de polynômes linéaires $\left(1+b_{\alpha} X\right)_{\alpha \in I} \subset F(X)^{\times}$est $\mathbb{F}_{2}$-linéairement indépendante modulo $F(X)^{\times 2}$. D'un autre côté, tous ces polynômes deviennent des carrés dans $F((X))$, donc aussi dans $F\langle X\rangle$.

Notons $F_{*}$ le sous-corps $F(X)\left(\sqrt{\left(1+b_{\alpha} X\right)\left(1+b_{\alpha^{\prime}} X\right)}\right)_{\alpha, \alpha^{\prime} \in I}$ de $F\langle X\rangle$ et posons $r:=\left(1+b_{\alpha_{0}} X\right)$ pour $\alpha_{0} \in I$ arbitraire. Pour tout $b \in F^{\times}$on a $r \in D_{F_{*}}(\langle 1, b X\rangle)$, puisque $b F^{\times^{2}}=b_{\alpha} F^{\times^{2}}$ pour $\alpha:=b F^{\times^{2}} \in I$, et ainsi $r \cdot\left(1+b_{\alpha} X\right) \in F_{*}^{\times^{2}}$. 
L'élément $r$ n'est pas un carré dans $F_{*}(\sqrt{X})$. Posons $L:=F\langle X\rangle(\sqrt[\infty]{X})$. Nous choisissons $K$ comme extension de $F_{*}(\sqrt{X})$ dans $L$ maximale pour la propriété que $r$ ne soit pas un carré dans $K$. Alors $K / F(X)$ est une 2extension et l'extension $K / F$ est de degré de transcendance 1. D'après (8.3), toute forme quadratique anisotrope sur $F$ reste anisotrope sur $L$, donc aussi sur $K$. Par choix, $K$ admet une seule extension quadratique dans $L$, à savoir $K(\sqrt{r})$. Comme $L^{\times}=F^{\times} L^{\times 2}$ d'après (8.3), il vient $K^{\times}=\left(F^{\times} \cup r F^{\times}\right) K^{\times 2}$.

Déjà sur $F_{*}(\sqrt{X})$, toute forme binaire $\langle 1, b\rangle$ avec $b \in F^{\times}$représente $r$. Il vient $F^{\times} \subset D_{K}(\langle 1,-r\rangle) \ni-r$, d'où $D_{K}(\langle 1,-r\rangle)=K^{\times}$. Comme la forme $\langle 1,-r\rangle$ devient hyperbolique sur $L$, elle ne provient pas d'une forme définie sur $F$. Cela montre que $r \in \mathrm{R}(K) \backslash F^{\times} K^{\times 2}$.

Pour $x \in \mathrm{R}(F)$ on a bien $K^{\times}=\left(F^{\times} \cup r F^{\times}\right) K^{\times 2} \subset D_{K}(\langle 1,-x\rangle)$, donc $x \in \mathrm{R}(K)$. On en déduit que $(\mathrm{R}(F) \cup r \mathrm{R}(F)) K^{\times 2} \subset \mathrm{R}(K)$.

Pour montrer l'inclusion réciproque, considérons un élément $x$ de $\mathrm{R}(K)$. Comme $K^{\times}=\left(F^{\times} \cup r F^{\times}\right) K^{\times 2}$ on peut choisir $y \in K^{\times 2} \cup r K^{\times 2}$ tel que $x y \in F^{\times}$. Ainsi $y \in R(K)$ et par suite $D_{K}(\langle 1,-x y\rangle)=D_{K}(\langle 1,-x\rangle)=K^{\times}$. Pour tout $z \in F^{\times}$la forme $\langle 1,-x y, z\rangle$ devient isotrope sur $K$, elle est donc déjà isotrope sur $F$. Cela montre que $\langle 1,-x y\rangle$ est universelle sur $F$, i.e. $x y \in \mathrm{R}(F)$ et donc $x \in(\mathrm{R}(F) \cup r \mathrm{R}(F)) K^{\times 2}$.

8.5 Corollaire. Soient $F$ un corps de caractéristique différente de 2 et $m$ un entier positif. Il existe une extension $K / F$ de degré de transcendance $m$ telle que toute forme quadratique anisotrope sur $F$ reste anisotrope après extension des scalaires à $K$ et telle que l'on a les égalités $K^{\times}=F^{\times} \mathrm{R}(K)$, $\mathrm{R}(F)=F^{\times} \cap \mathrm{R}(K)$ et $\left|\mathrm{R}(K) / \mathrm{R}(F) K^{\times 2}\right|=2^{m}$.

Démonstration : On raisonne par récurrence sur $m$. Le cas $m=1$ s'obtient à partir du théorème (8.4) et fournit le pas de récurrence.

Remarque : Dans la situation du corollaire, si $F$ est sans radical alors $\left|\mathrm{R}(K) / K^{\times 2}\right|=2^{m}$.

8.6 Corollaire. On garde les notations du dernier corollaire. Pour qu'une forme quadratique sur $K$ provienne d'une forme quadratique définie sur $F$ il faut et il suffit que son discriminant appartienne $\grave{a} F^{\times} K^{\times 2}$. L'homomorphisme canonique d'extension des scalaires donne lieu à une suite exacte

$$
0 \longrightarrow W(F) \longrightarrow W(K) \longrightarrow(\mathbb{Z} / 2 \mathbb{Z})^{m} \longrightarrow 0 .
$$


Démonstration : Soit $\psi$ une forme quadratique de dimension $n$ sur $K$. On écrit $\psi \cong\left\langle a_{1} r_{1}, \ldots, a_{n} r_{n}\right\rangle$ avec $a_{1}, \ldots, a_{n} \in F^{\times}$et $r_{1}, \ldots, r_{n} \in \mathrm{R}(K)$. Il suit de (4.1) que tout élément du radical est un facteur de similitude de toute forme binaire. Il vient donc $\psi \cong\left\langle a_{1}, \ldots, a_{n-1}, r a_{n}\right\rangle$ avec $r:=r_{1} \cdots r_{n}$. Le discriminant de $\psi$ est dans $F^{\times} K^{\times 2}$ si et seulement si $r \in F^{\times} K^{\times^{2}}$; si tel est le cas alors $\psi$ provient d'une forme définie sur $F$. Réciproquement, si $\psi$ provient d'une forme définie sur $F$ alors il est clair que $d_{ \pm}(\psi) \in F^{\times} K^{\times 2}$.

L'homomorphisme $W(F) \longrightarrow W(K)$ est injectif puisque toute forme quadratique anisotrope sur $F$ reste anisotrope sur $K$. D'après la première partie de la preuve, son image est égale au noyau de l'homomorphisme surjectif $W(K) \longrightarrow K^{\times} / F^{\times} K^{\times^{2}}$ induit par le discriminant. Les propriétés de l'extension $K / F$ permettent d'obtenir

$$
K^{\times} / F^{\times} K^{\times 2}=F^{\times} \mathrm{R}(K) / F^{\times} K^{\times 2} \cong \mathrm{R}(K) / \mathrm{R}(K) \cap F^{\times} K^{\times 2}=\mathrm{R}(K) / \mathrm{R}(F) K^{\times 2}
$$

où le dernier quotient est un groupe abélien 2-élémentaire de cardinal $2^{m}$.

8.7 Question. Étant donné un corps $F$, existe-t-il toujours une extension $K / F$ telle que $K$ soit sans radical, telle que $K^{\times}=F^{\times} K^{\times 2}$ et telle que toute forme quadratique anisotrope sur $F$ et de dimension au moins 3 reste anisotrope après extension des scalaires $\grave{a} K$ ?

Remarque : On ne rend pas le problème plus difficile en exigeant en plus que $K / F$ soit une 2-extension. En effet, si $K / F$ est une extension qui répond positivement à la question et si $K^{\prime}$ désigne la clôture quadratique de $F$ dans $K$ alors $K^{\prime} / F$ est une 2-extension qui convient aussi.

8.8 Proposition. Soit $H \subset F^{\times}$. On pose $K:=F(\sqrt{H})$. Pour que $\operatorname{Br}(K / F)=0$ il faut et il suffit que $H \subset \mathrm{R}(F)$.

Démonstration : S'il existe $h \in H \backslash \mathrm{R}(F)$ alors on peut trouver $x \in F^{\mathrm{x}}$ tel que la $F$-algèbre de quaternions $(h, x)_{F}$ représente une classe non triviale dans $\operatorname{Br}(K / F)$.

Supposons maintenant que $H \subset R(F)$ et montrons que $\operatorname{Br}(K / F)=0$. Il suffit d'examiner le cas où $H$ est fini. On procède par récurrence sur le cardinal de $H$. Si $H=\emptyset$, c'est clair. Sinon, choisissons $h \in H$ et posons $F^{\prime}:=F(\sqrt{h})$ et $H^{\prime}:=H \backslash\{h\} ;$ alors $K=F^{\prime}\left(\sqrt{H^{\prime}}\right)$. Puisque $h \in \mathrm{R}(F)$ on a $\operatorname{Br}\left(F^{\prime} / F\right)=0$. Le théorème (6.1) assure que $\mathrm{R}(F) \subset \mathrm{R}\left(F^{\prime}\right)$; alors $H^{\prime} \subset \mathrm{R}\left(F^{\prime}\right)$ et par hypothèse de récurrence on obtient $\operatorname{Br}\left(K / F^{\prime}\right)=0$. Il vient $\operatorname{Br}(K / F)=0$. 
8.9 Théorème. Pour tout corps $F$ de caractéristique différente de 2 on définit $F_{\mathrm{red}}:=\bigcup_{i \geq 0} F_{i}$ avec $F_{0}:=F$ et $F_{i}:=F_{i-1}\left(\sqrt{\mathrm{R}\left(F_{i-1}\right)}\right)$ pour $i \geq 1$. Alors $F_{\text {red }} / F$ est une 2-extension qui vérifie les propriétés suivantes:

(a) $\operatorname{Br}\left(F_{\text {red }} / F\right)$ est trivial,

(b) $F_{\text {red }}$ est sans radical,

(c) si $K / F$ est une 2-extension de $F$ telle que $K$ est sans radical alors $K$ contient $F_{\text {red, }}$

(d) si $F^{\prime}$ est un corps tel que $F \subset F^{\prime} \subset F_{\text {red }}$ alors $F_{\text {red }}$ et $F_{\text {red }}^{\prime}$ coinncident,

(e) l'homomorphisme $\mathrm{R}(F) \rightarrow W(F)$ défini dans (4.4) et l'homomorphisme canonique $W(F) \rightarrow W\left(F_{\mathrm{red}}\right)$ forment une suite exacte

$$
\mathrm{R}(F) \longrightarrow W(F) \longrightarrow W\left(F_{\text {red }}\right) .
$$

Démonstration: (a) La proposition (8.8) assure que $\operatorname{Br}\left(F_{i} / F_{i-1}\right)=0$ pour tout $i \geq 1$. Comme $F_{0}=F$, il s'ensuit que $\operatorname{Br}\left(F_{i} / F\right)=0$ pour tout $i \geq 1$, puis que $\operatorname{Br}\left(F_{\text {red }} / F\right)=0$.

(b) Soit $x \in F_{\text {red }}^{\times} \backslash F_{\text {red }}^{\times}{ }^{2}$. Alors il existe $i \geq 0$ tel que $x \in F_{i}$. Comme $x$ ne devient pas un carré dans $F_{i+1}$, on sait que $x \notin \mathrm{R}\left(F_{i}\right)$. Il existe donc $y \in F^{\times}$ tel que la $F_{i}$-algèbre de quaternions $(x, y)_{F_{i}}$ est à division. Par construction on a $\left(F_{i}\right)_{\text {red }}=F_{\text {red }}$, alors, d'après $(\mathrm{a}), \operatorname{Br}\left(F_{\text {red }} / F_{i}\right)=0$. Par suite, $(x, y)_{F_{\text {red }}}$ est à division, d'où $x \notin \mathrm{R}\left(F_{\text {red }}\right)$.

(c) Soit $K / F$ une 2-extension telle que $\mathrm{R}(K)=K^{\times 2}$. Pour montrer que $F_{\text {red }} \subset K$ il suffit de voir que $F_{i} \subset K$ pour tout $i \geq 0$. On raisonne par récurrence sur $i$. Soit $i \geq 0$ tel que $F_{i} \subset K$. Alors $K / F_{i}$ est une 2-extension, et (6.2) affirme que $\mathrm{R}\left(F_{i}\right) \subset \mathrm{R}(K)=K^{\times^{2}}$. Il s'ensuit que $F_{i+1} \subset K$.

(d) C'est une conséquence directe de (b) et de (c).

(e) Toute forme $\langle 1,-r\rangle$ avec $r \in \mathrm{R}(F)$ devient hyperbolique sur $F_{1}$, donc sur $F_{\text {red }}$. Par conséquent, la composition $\mathrm{R}(F) \rightarrow W(F) \rightarrow W\left(F_{\text {red }}\right)$ est triviale. Pour mettre en évidence l'exactitude en $W(F)$, on va montrer que les seules formes anisotropes sur $F$ qui deviennent isotropes sur $F_{\text {red }}$ sont les $\langle 1,-r\rangle$ avec $r \in F^{\times} \backslash \mathrm{R}(F)$.

Soit $\varphi$ une forme anisotrope sur $F$ qui devient isotrope sur $F_{\text {red. }}$. Choisissons un sous-corps $K$ de $F_{\text {red }}$ contenant $F$, maximal pour la condition que $\varphi_{K}$ soit anisotrope. Alors $K \neq F_{\text {red }}$ et, par (2.6), $K$ admet une extension quadratique $K(\sqrt{x})$, pour un certain $x \in K^{\times}$, contenue dans $F_{\text {red. }}$. Par choix de $K, \varphi_{K(\sqrt{x})}$ est isotrope. D'après (d) on a $K_{\text {red }}=F_{\text {red. }}$ La partie (a) appliquée au corps $K$ montre alors que $\operatorname{Br}\left(F_{\text {red }} / K\right)=0$. Par 
conséquent, $\operatorname{Br}(K(\sqrt{x}) / K)$ est trivial, ce qui revient à dire que la forme binaire $\langle 1,-x\rangle$ sur $K$ est universelle (3.1). Comme $\varphi_{K}$ est anisotrope et $\varphi_{K(\sqrt{x})}$ isotrope, $\varphi_{K}$ contient une sous-forme semblable à $\langle 1,-x\rangle[13$, Chap. VII, Lemma 3.1.]. Puisque la forme $\langle 1,-x\rangle$ est universelle et multiplicative et $\varphi_{K}$ anisotrope, on obtient $\varphi_{K} \cong\langle 1,-x\rangle$. La forme $\varphi$ étant définie sur $F$ il vient $x \in F^{\times} K^{\times^{2}}$. On peut donc supposer que $x \in F^{\times}$. À l'aide de (a), on obtient

que $\mathrm{R}(F)=F_{\text {red }}^{\times} \cap F^{\times} \ni x$. Ainsi la forme $\langle 1,-x\rangle$ est universelle sur $F$. Par le même raisonnement que ci-dessus, on en déduit que $\varphi \cong\langle 1,-x\rangle$ sur $F$.

\section{Références}

[1] Lawrence Berman. Pythagorean fields and the Kaplansky radical. $J$. Algebra, 61(2):497-507, 1979.

[2] N. Bourbaki. Éléments de mathématique. Algèbre. Chapitres 1 à 3 . Hermann, Paris, 1970.

[3] Craig M. Cordes. Kaplansky's radical and quadratic forms over non-real fields. Acta Arith., 28(3):253-261, 1975/76.

[4] Richard Elman and T. Y. Lam. On the quaternion symbol homomorphism $g_{F}: k_{2} F \rightarrow B(F)$. Lecture Notes in Math., Vol. 342:447-463, 1973.

[5] Richard Elman and T. Y. Lam. Quadratic forms and the $u$-invariant. I. Math. Z., 131:283-304, 1973.

[6] Richard Elman and T. Y. Lam. Quadratic forms under algebraic extensions. Math. Ann., 219(1):21-42, 1976.

[7] Tatsuo Iwakami, Daiji Kijima, and Mieo Nishi. Kaplansky's radical and Hilbert Theorem 90. III. Hiroshima Math. J., 15(1):81-88, 1985.

[8] Irving Kaplansky. Fröhlich's local quadratic forms. J. Reine Angew. Math., 239/240:74-77, 1969.

[9] Daiji Kijima and Mieo Nishi. Kaplansky's radical and Hilbert theorem 90. Hiroshima Math. J., 11(2):443-456, 1981.

[10] Daiji Kijima and Mieo Nishi. Kaplansky's radical and Hilbert Theorem 90. II. Hiroshima Math. J., 13(1):29-37, 1983. 
[11] Mieczysław Kula. Fields with prescribed quadratic form schemes. Math. Z., 167(3):201-212, 1979.

[12] Mieczysław Kula. Fields with nontrivial Kaplansky's radical and finite square class number. Acta Arith., 38(4):411-418, 1980/81.

[13] Tsit Yuen Lam. The algebraic theory of quadratic forms. W. A. Benjamin, Inc., Reading, Mass., 1973. Mathematics Lecture Note Series.

[14] O. T. O'Meara. Introduction to quadratic forms. Springer-Verlag, New York, 1971.

[15] Richard S. Pierce. Associative algebras. Springer-Verlag, New YorkHeidelberg-Berlin, 1982. Graduate Texts in Mathematics, 88.

[16] Louis H. Rowen. Ring theory. Vol. II. Academic Press Inc., Boston, MA, 1988.

[17] David J. Saltman. Division algebras over p-adic curves. J. Ramanujan Math. Soc., 12(1):25-47, 1997.

[18] Winfried Scharlau. Quadratic and Hermitian forms. Springer-Verlag, Berlin, 1985.

[19] Kazimierz Szymiczek. Quadratic forms over fields with finite square class number. Acta Arith., 28(2):195-221, 1975/76.

[20] Jean-Pierre Tignol. Réduction de l'indice d'une algèbre simple centrale sur le corps des fonctions d'une quadrique. Bull. Soc. Math. Belg. Sér. A, 42(3):735-745, 1990.

[21] Joseph L. Yucas. Quadratic forms and radicals of fields. Acta Arith., 39(4):313-322, 1981. 